\title{
Human Intrusion and Motion Detection System
}

\author{
Kunal Deo \\ NMIMS, MPSTME, MUMBAI
}

\author{
Rishi Deedwania \\ NMIMS, MPSTME, MUMBAI
}

\author{
Swati Bairagi \\ NMIMS, MPSTME, MUMBAI
}

\begin{abstract}
Human intrusion and motion detection system can detect the presence of a human being using sensors. When a human is detected it takes pictures or videos using a camera according to the given setting. This is then sent via email to the owner of the system and a Whatsapp text alert is sent simultaneously. This system also interface other security systems that can alert a person present in the house of an intrusion, in this model a buzzer is interfaced, an LED light and a camera to a Raspberry Pi $3 b$ plus, using a PIR sensor for detection for a very basic model of our proposed security system
\end{abstract}

\section{General Terms}

PIR Sensors, Security, Human detection

\section{Keywords}

Raspberry Pi, Surveillance system, Alerting system

\section{INTRODUCTION}

Nowadays Home Security is a major concern. Existing home security systems although technologically advanced are still ineffective. Burglars have found ways to work around existing security systems and disable them as they tend to know the placements of the sensors, while majority of detection by burglar alarms are false positives and existing systems are also very expensive. ed by us in this paper aims to make an easily modifiable home security system using PIR sensors at its base while also being affordable.

\section{RELATED WORK}

Detecting the presence of human beings can be done using many methods. One method is interfacing of a camera with a Raspberry Pi and is using a PIR sensor to find the direction of movement while offering privacy both ways, a video feed for surveillance is also included and received through a smartphone [1]. The second technique to accurately detect only human beings is by combining a PIR sensor with a seismic sensor which will result in more accurate detection of humans and not other animals [2]. In another technique, the detection is combined with a video feed and sound detection resulting in more accurate detection of humans as well [3]. The detailed operation of the PIR sensors, details about Fresnel lenses and the effect of temperature on the PIR sensors, which is useful when taking into consideration the environments the systems operate in[4][6]. The detection of Motion by PIR sensor using the RST paradigm results in more accurate pinpointing the location of an object[5]. Mounting the sensors onto the walls and designing a system with multiple sensors at different locations [7]. Methods of avoiding flora and fauna detection to decrease number of false positives[8]. Methods of accurate detection by PIR sensor using SVM and HOG techniques [9]

\section{WORKING PRINCIPLE}

The working of the PIR sensors is complex. Because there are multiple variables affecting the input and output. The PIR sensors detect changes in IR radiations within their FOV. Whenever an object

passes in the field of view (FOV) of the PIR sensor, the temperature of the area will change from the current temperature to that of the temperature of the body which the sensor detects as amount of IR radiation change.

Upon detection the PIR sensor notifies the Raspberry Pi which in turn takes a picture using the camera connected to it and triggers the buzzer and LED lights. The flow of the system is as follows ,

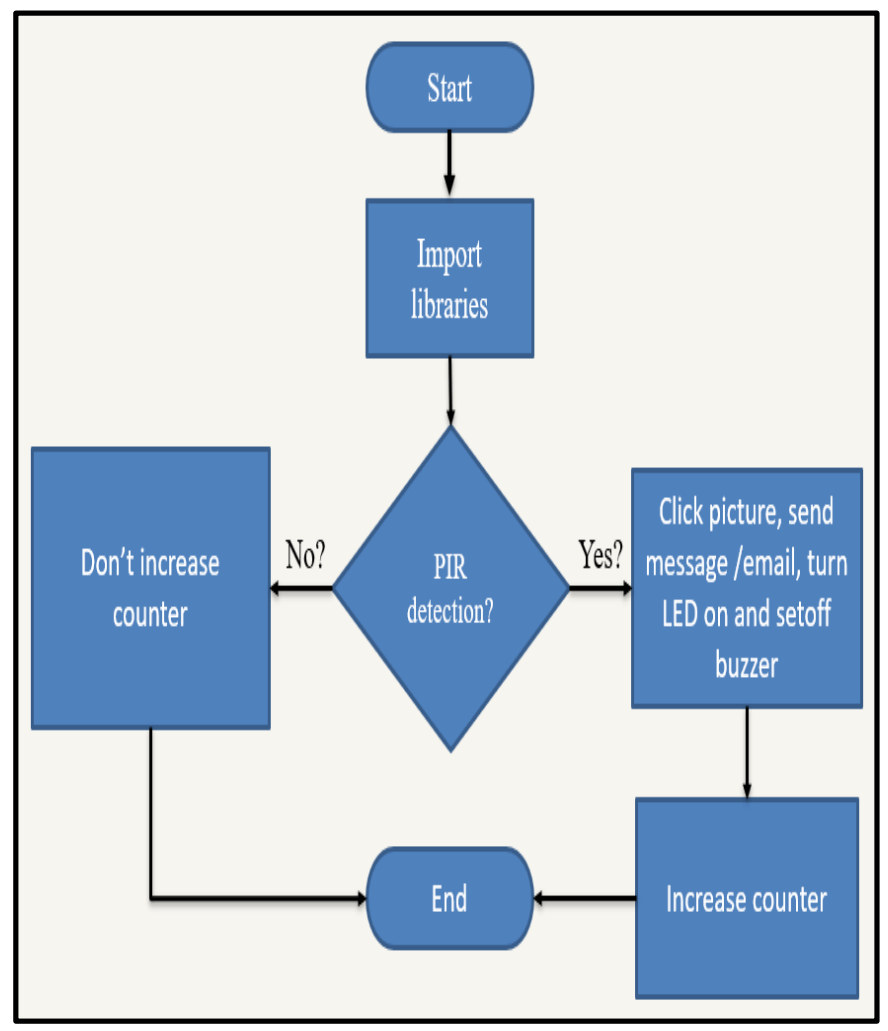

Fig 1 Flow chart of the process of human intrusion detection

The flow chart shows the generalized logic of the system. The prototype model is built using a PIR sensor and the Raspberry Pi is used to inter face the sensor to the other security devices Though this system can be generalized for any for any sensor or a set of sensors. This can be represent in the block diagram given below, 


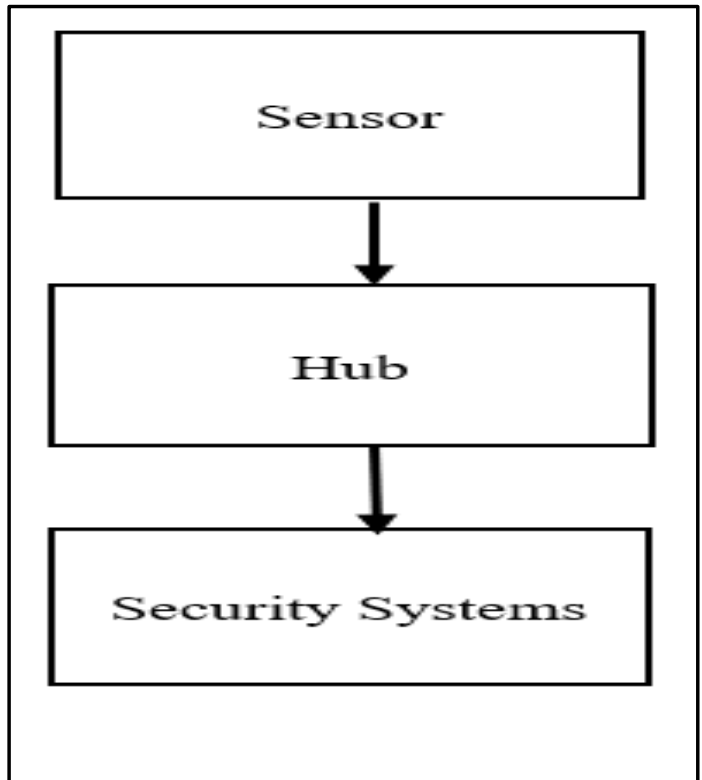

Fig 2 Block Diagram of the Human intrusion detection system

For a more generalized system as shown in the block diagram, many different sensors can be connected in a network. These sensors can feed different types of input to the main hub which will then process the information and give a result accordingly. For human detection Infrared sensors can be combined with seismic sensors to feed into the hub. Humans have specific infrared radiation wavelength (10 microns) and seismic sensors which can be programmed to detect human footsteps. This will give a far more accurate result than using one sensor. Similarly using the block diagram better alerting mechanisms can be devised. A network of Cameras and Alarms can be also be set up along with the sensor network.

The network topology used in this model is inspired by a star topology with the hub (in this system the Raspberry Pi) Connected to multiple devices. This ensures that even if one device is disabled it will not render the others ineffective.

\section{FUNCTIONAL DESCRIPTION}

\subsection{PIR Sensors}

It detects IR radiation emitted by bodies, so it can detect objects emitting heat and temperature changes. Fresnel lenses increase the range of the sensors. These lenses are made up of materials which help capture the radiation from the Infrared spectrum. Unlike Active IR sensors PIR sensors only detect objects when in motion. Its sensitivity can be adjusted using the knobs.

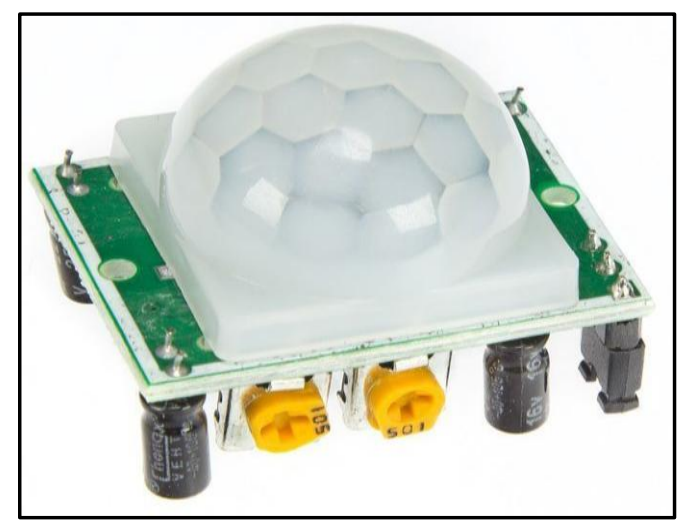

Fig 4.1: A picture of a PIR sensor

\subsection{Raspberry Pi 3b Plus}

In this model a Raspberry Pi 3B plus is used. This Raspberry PI has a 64-bit quad core processor running at $1.4 \mathrm{GHz}$.

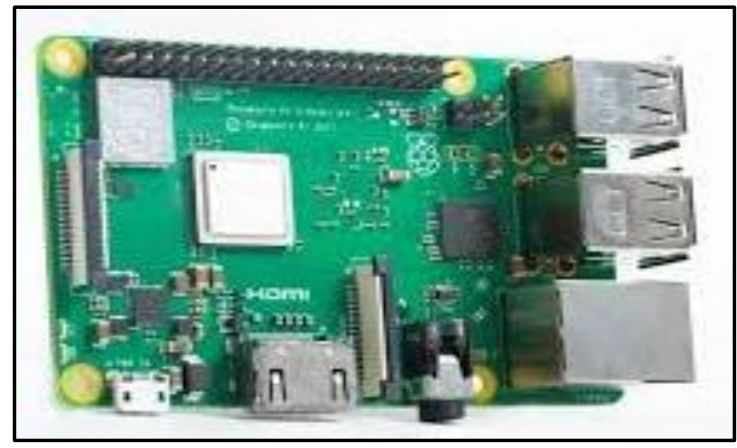

Fig 4.2: a picture of a Raspberry Pi 3b Plus

\subsection{Pi Camera}

The $\mathrm{Pi}$ Camera is 5 Megapixels, it has dimensions of $25 \times 24 \times 9 \mathrm{~mm}$ and has a focal length of $3.60+/-0.01 \mathrm{~mm}$

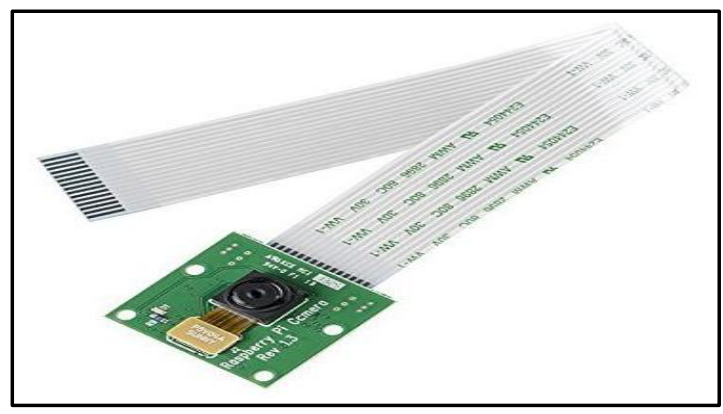

Fig 4.3: A Raspberry Pi camera

\subsection{Buzzer}

A buzzer is an electrical device whose purpose alert people using sound.

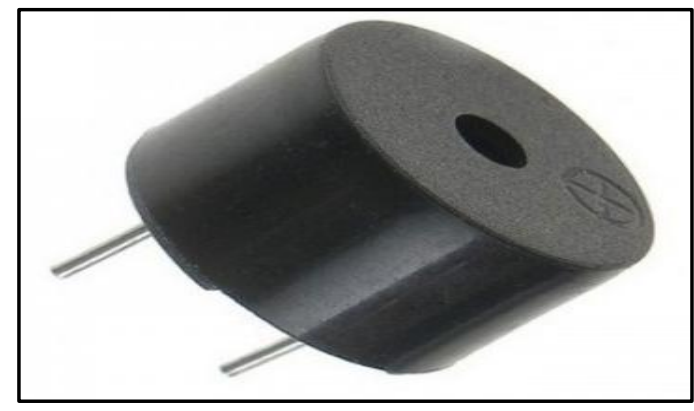

Figure 4.4: Picture of an electrical buzzer

\subsection{LED light}

An LED light acts as a visual signaling device

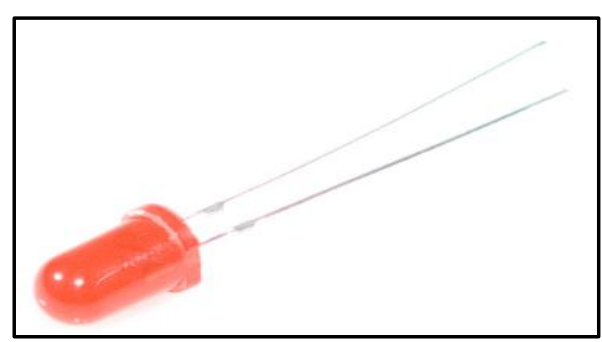

Fig 4.5: Picture of an LED light 


\section{RESULTS AND DISCUSSION}

The Circuit made is a simplified representation of the theoretical model represented by the block diagram.

The circuit only uses one sensor which is a PIR sensor. A buzzer and an LED are used to represent devices which will alert users to the presence of an intruder. he common ground pin for all devices is pin number 6 . For the PIR sensor The Vcc voltage is provided by pin 2 which is a $5 \mathrm{~V}$ pin while the input is given at GPIO 3 which is pin 5

The output given to the buzzer is given through the GPIO 24 which is pin 18 .

The output of the LED is given through GPIO 18. The input of the 5 Mega pixel Raspberry Pi Camera goes to Pi Camera Slot

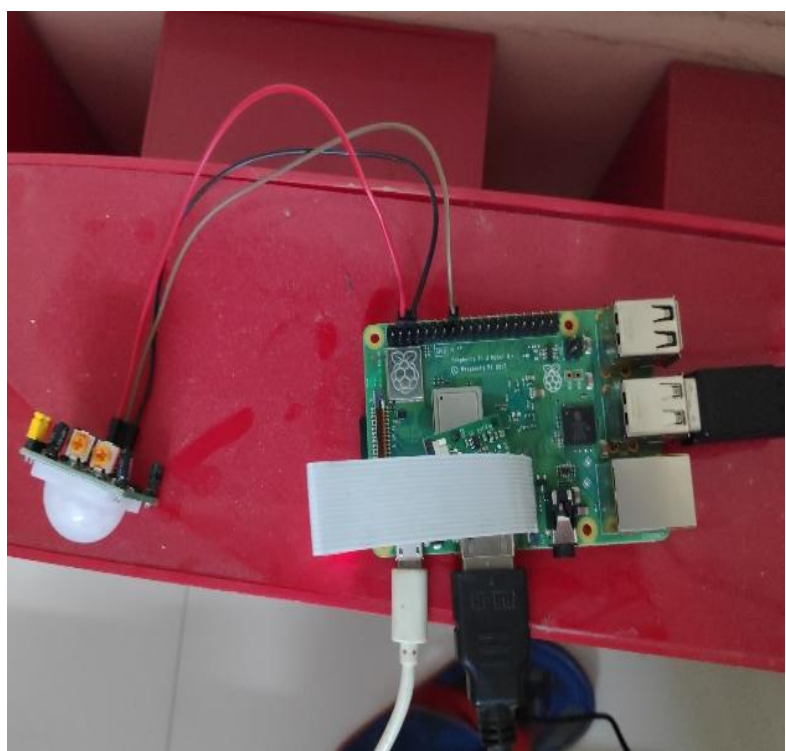

Fig 5.1. PIR sensor and PI Camera interface

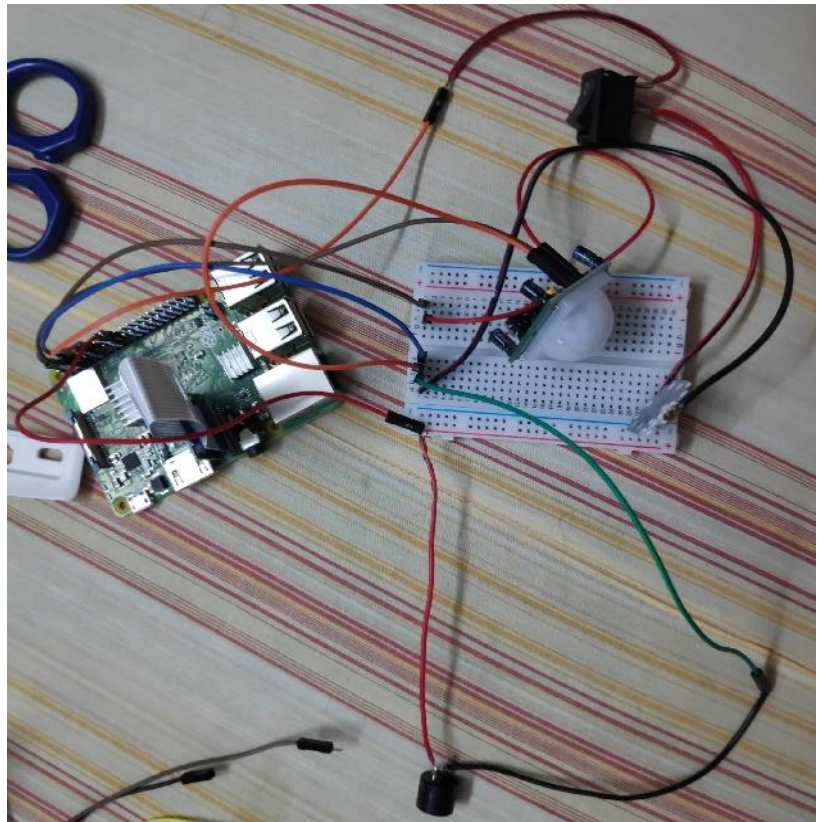

Fig 5.2 buzzer, PIR sensor and LED interface

The message upon detection is sent via Whatsapp and is received along with a picture taken via the camera which is sent through email

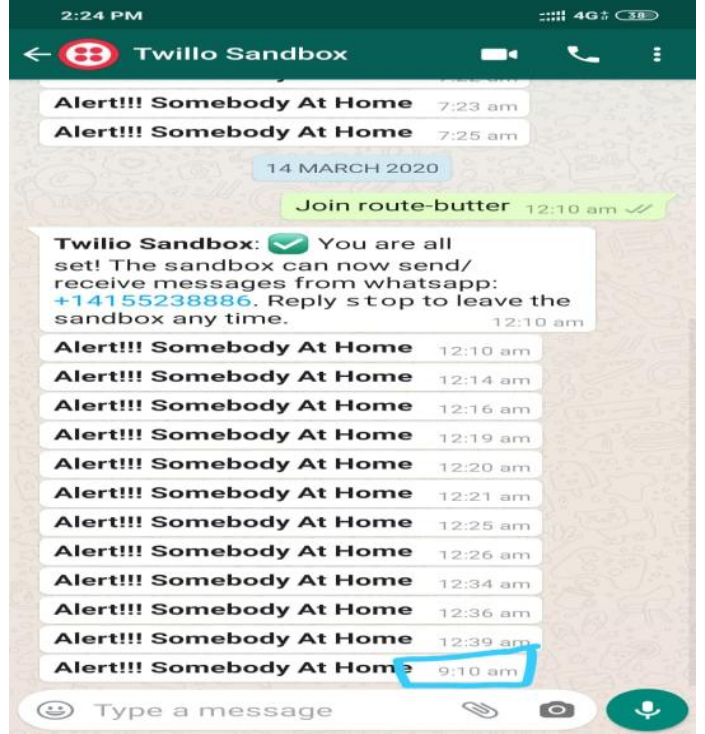

Fig 5.3 Text message for intruder detection
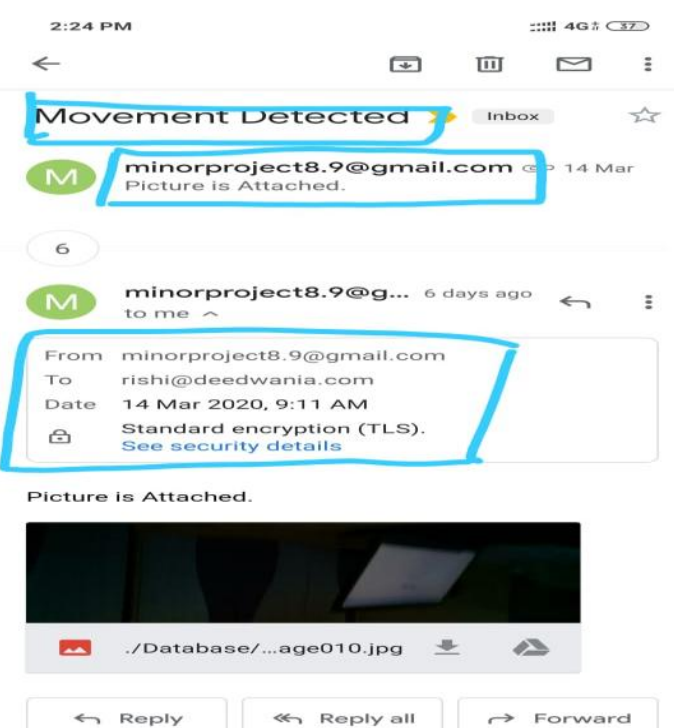

Fig 5.4 Email of image taken for intruder detection

\section{CONCLUSION}

Human intrusion and motion detection system is meant to provide a versatile framework using which a security system can be set up according to the need and situation. This system is of a very rudimentary design. It is more affordable than existing security systems and modifiable

This system can be easily improved upon by adding more security devices or improving method of communication with devices.

The disadvantage that the system possesses is that it is based on a star topology network and if the central hub is disabled through any means it will cause the entire system to collapse

Another disadvantage of the current system is the usage of a single sensor to detect a human, which although more accurate than an active IR sensor in human detection could still give false positives.

A simple solution to overcome this would be to combine a network of sensors to collect enough data to make sure the detected object is a human. 


\section{FUTURE SCOPE}

This system can be further be modified with the use of the concept of Internet of things. The change in the system will only be the communication medium which will change from wired to wireless. This security system can be connected to a common net which will directly alert law enforcement in case of an intrusion. Due to its versatility and ease of setting

up the system can also be used for setting up fast, temporary and modifiable surveillance systems making it effective for applications where permeant surveillance set ups are not needed one example being security in conflict zones.

\section{REFERENCES}

[1] Sanjana Prasad, P.Mahalakshmi, A. John Clement Sunder and R.Swathi, "Smart surveillance monitoring system using Raspberry Pi and PIR sensor", International Journal of Computer Science and Information Technologies, Vol. 5 (6), December 2014.

[2] Guodong Feng, Xuemei Guo and Guoli Wang, "Infrared Motions sensing system for human following", Sensors and Actuators volume 185, October 2012.

[3] Mohit Bais, Kanupriya Madan, Ankit Bharti and Prof. Prity Yadav, "Alive Human Detection Robot", International Journal of Scientific Research in Computer Science, Engineering and Information Technology volume 2 issue 1, January-February 2017.
[4] Ajay Kumar Tiwari, Prince Raj, Justice Kumar and Mr.Ashish Tiwary, "Motion detection using PIR sensor", International Journal of Scientific Development and Research(IJSDR) volume 1 issue 5, May 2016.

[5] Sarvana Kumar K, Priscilla $P$ and Germiya K Jose, "Human Detection Robot using PIR sensors", International Journal of Science, Engineering and Technology Research (IJSETR)Volume 4, Issue 3, March 2015.

[6] Yogesh Pawar, Abhay Chopde and Mandar Nandre, "Motion Detection Using PIR Sensor", International Research Journal of Engineering and Technology, Volume: 05 Issue: 04, April 2018.

[7] M. Moghavvemi and Lu Chin Seng. "pyroelectric infrared sensor for intruder detection" in TENCON 2004. 2004 IEEE Region 10 Conference (Volume:4 ), pp. 656 - 659, 2004

[8] Tarun Choubisa, Raviteja Upadrashta, Sumankumar Panchal, A. Praneeth, Ranjitha H. V. and Kaushik Senthoor in "Who Goes There? A PIR-Sensor-Based Intrusion Classification System for an Outdoor Environment" in LCN 2016, December 2016

[9] Nico Surantha and Wingky R. Wicaksono in "An IoT based House Intruder Detection and Alert System using Histogram of Oriented Gradients" in Journal of Computer Science 2019, issue 08, August 2019 